\section{Genetic nephrology}

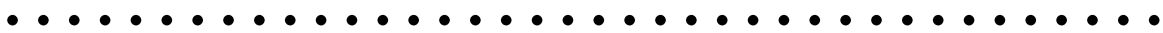

The genetics of renal disease

Edited by Frances Flinter, Eamonn Maher, Anand Saggar-Malik. Oxford University Press, New York, 2003. pp. 568. $£ 120.00$ (hardback). ISBN 0192631462.

C.............

Cristina Rusu

European Journal of Human Genetics (2005) 13, 891.

doi:10.1038/sj.ejhg.5201416

$\mathrm{T}$

he authors have achieved an outstanding book on the genetics of renal disorders. This subject was first approached in The Genetics of Renal Tract Disorders by MD'A Crawfurd (1988). There have been major changes in our knowledge of medical genetics since then, many genetic factors involved in the pathogenesis of a wide range of genetic diseases being described meanwhile. Frances Flinter, Eamonn Maher and Anand Saggar-Malik are to be complimented for having undertaken the task to achieve a second monograph on this subject, entitled The Genetics of Renal Disease. The book offers much more than a simple review of genetic implications in renal disorders. It is a multiauthor approach, gathering the opinions of the most important specialists in the field. The material is structured in 24 chapters, covering not only basic notions (renal function, renal development and kidney and urinary tract malformations), disorders with major renal involvement (chromosomal disorders, dysmorphic syndromes, Alport syndrome, polycystic kidney disease, metabolic diseases, disorders of tubular transport, tuberous sclerosis, neurofibromatosis and Bardet-Biedl syndrome) but also systemic disorders affecting the kidney. The last five chapters refer to oncologic implications (Wilms tumour, Von Hippel-Lindau disease and inherited predispositions to kidney cancer) and to gene therapy in renal disease and renal cancer. Every chapter is a state-of-art presentation including pathogenesis (with comprehensive yet accessible molecular mechanisms), suggestive clinical features and management. Pathogenic mechanisms include all the genes involved, making the material useful both for practitioners and for scientists. Animal models are provided for different disorders to complete pathogenic mechanisms. The explanations are beautifully illustrated with plates and schemes that make the lecture very pleasant. For those looking for a specific topic, the alphabetic index proves very useful.

Most interesting to me were the chapters on renal development and kidney and lower urinary tract malformations. They offer a clear and adequately illustrated overview of genes and factors involved in renal and urinary tract development. For those who want to understand the mechanisms that lead to renal and urinary malformations in order to ask for the proper investigations and to achieve a correct management of the patient, all this information come in useful.

Gene therapy is another attractive part. The authors refer to the most important strategies and include adequate plates that illustrate the mechanisms, especially for renal cancer. Maybe the next edition will include more information referring to gene therapy in other disorders and other methods of treatment.

The chapters regarding specific renal/ urinary tract disorders are very interesting and useful for the practitioner, due to plenty of practical elements such as the renal chromosomal map, chromosomal implications of fetal renal anomalies detected at ultrasonography. There are also included a table with syndromes of specific renal abnormalities, genes and molecular mechanisms involved in primary hereditary nephropathies, renal manifes- tations in metabolic disorders, genetic bases of stone-forming disorders, clinical and biochemical features of salt wasting disorders and primary acidopathies, genetics of Bardet-Biedl syndrome, genetics of glomerulonephritis and systemic disorders affecting the kidney and many more.

Another interesting chapter is that referring to complex renal diseases like glomerulonephritis and systemic disorders affecting the kidney. The genetic data involved in the pathogenesis of these complex disorders are elegantly presented here. It is surprising how disorders considered classically as having an immunologic determinism become more and more genetic. Identifying genes and molecular pathways that lead to nephropathy in complex disorders like systemic lupus erythematosus or diabetes mellitus will represent a major challenge of the research teams working in this field.

Many monographs are already out of date when they are published due to the rapid development of knowledge in the field of genetics. This is not valid for the present book, that due to the care and efforts of the authors have most of the bibliographical references dated 2001-2002 and even 2003. Very useful from the practical point of view are the Internet addresses that complete the bibliography for some chapters.

In summary, this comprehensive yet accessible presentation admirably joins together genetic elements, pathogenic pathways, clinical features and management directions. Those who look for a specific disorder can use the index and every chapter could be read selectively or completely. The text is completed with plates and schemes that illustrate the most important ideas. All together, this monograph is highly recommended for clinicians that take care of patients with renal disorders like pediatricians, internal physicians, nephrologists, urologists and clinical geneticists and residents training for these specialties, as well as for scientists

Cristina Rusu is at the Department of Human Genetics, University of Medicine and Pharmacy, Str Universitatii 16, Iasi 700115, Romania. E-mail: abcrusu@yahoo.com 